\title{
Immune dysregulation as a driver of idiopathic pulmonary fibrosis
}

\author{
Kevin Shenderov, ${ }^{1,2}$ Samuel L. Collins, ${ }^{1,2}$ Jonathan D. Powell, ${ }^{2}$ and Maureen R. Horton ${ }^{1}$ \\ 'Department of Medicine, Division of Pulmonary and Critical Care, Johns Hopkins University School of Medicine, Baltimore, Maryland, USA. ${ }^{2}$ Bloomberg-Kimmel Institute for Cancer Immunotherapy, \\ Sidney Kimmel Comprehensive Cancer Research Center, Department of Oncology, Johns Hopkins University School of Medicine, Baltimore, Maryland, USA.
}

\begin{abstract}
Idiopathic pulmonary fibrosis (IPF) affects hundreds of thousands of people worldwide, reducing their quality of life and leading to death from respiratory failure within years of diagnosis. Treatment options remain limited, with only two FDA-approved drugs available in the United States, neither of which reverse the lung damage caused by the disease or prolong the life of individuals with IPF. The only cure for IPF is lung transplantation. In this review, we discuss recent major advances in our understanding of the role of the immune system in IPF that have revealed immune dysregulation as a critical driver of disease pathophysiology. We also highlight ways in which an improved understanding of the immune system's role in IPF may enable the development of targeted immunomodulatory therapies that successfully halt or potentially even reverse lung fibrosis.
\end{abstract}

\section{Introduction}

Major advances in understanding the immune system in recent decades have enabled the development of exciting therapies for a variety of diseases - from novel immunomodulators for autoimmune diseases to immune checkpoint inhibitors that have created a paradigm shift in the treatment of many cancers. Our grasp of the immune system's role in the pathophysiology of many pulmonary diseases remains limited, however. Further research in this area may lead to the development of immune-targeted therapies for diseases such as idiopathic pulmonary fibrosis (IPF).

Pulmonary fibrosis is not a single disease. Instead, it is the result of various processes that cause widespread scarring in the lungs. This scarring impedes the exchange of oxygen and carbon dioxide between the alveoli (air sacs) and pulmonary blood vessels, leading individuals with pulmonary fibrosis to experience shortness of breath, dry cough, a requirement for supplementary oxygen, and eventual respiratory failure if the condition progresses. Causes of lung fibrosis include autoimmune/connective tissue diseases (such as rheumatoid arthritis or scleroderma), drugs such as the antiarrhythmic amiodarone or the chemotherapeutic bleomycin, radiation, occupational exposures such as coal or silica, and some allergens (e.g., pigeon fancier's lung). An obvious etiology for fibrosis will guide treatment - for example, stopping the offending drug, avoiding the allergic or occupational exposure, or treating the underlying autoimmune disease with immunosuppressive medications. In many individuals, however, there is no discernible cause of fibrosis. These individuals are referred to as having idiopathic interstitial pneumonitis, the most common being IPF.

Authorship note: KS and SLC contributed equally to this work. Conflict of interest: The authors have declared that no conflict of interest exists. Copyright: (5) 2021, American Society for Clinical Investigation. Reference information: J Clin Invest. 2021;131(2):e143226. https://doi.org/10.1172/JCl143226.
IPF occurs primarily in individuals over 50 years of age, with two-thirds over 60 at the time of diagnosis, and more commonly in males than females (1). The prevalence of IPF has been estimated to be 10-20 per 100,000 individuals in the United States and Europe $(2,3)$, but the true epidemiology is difficult to determine because IPF initially presents with nonspecific symptoms, often delaying diagnosis for years. Treatment of this condition has been hampered by lack of understanding of disease pathophysiology and true natural history. There are only two FDA-approved medications for IPF - pirfenidone and nintedanib - and they do not stop or reverse disease progression or reduce mortality; they only modestly lower the rate of lung function worsening (4-6). These medications are also often difficult for patients to tolerate due to off-target effects leading to symptoms such as nausea, fatigue, and diarrhea. The only cure for IPF is lung transplantation. The pressing need for the development of novel therapies is reflected in the poor prognosis for individuals with IPF - the median survival after diagnosis was originally estimated to be only 3-5 years, although analyses of placebo groups in recent clinical trials and earlier observational studies suggest this may be an underestimate due to delays in diagnosis (7-10).

At its core, IPF can be thought of as a dysregulated wound healing response in the lung (11). Normal wound healing involves deposition of connective tissue such as collagen by fibroblasts at sites of injury. This is typically a self-limited local reaction, for example a laceration that heals with a small scar. In IPF, however, this wound healing response is widespread and continues over a prolonged time period, leading to diffuse deposition of connective tissue in the lung interstitium. It has been hypothesized that there is an inciting lung epithelial injury in IPF caused by environmental irritants such as cigarette smoke (smoking increases the risk of IPF), pollutants, or dust (12). Infection with herpesviruses such as EBV has also been suggested as a cause of epithelial injury because herpesvirus DNA has been found more frequently in the lungs of IPF patients than healthy controls (13). Damage to lung 
epithelial cells and extracellular matrix (ECM) leads to the release of cytokines, chemokines, and growth factors that induce fibroblast activation and proliferation. One major paradigm of disease pathophysiology proposes that aberrant interactions between epithelial cells and fibroblasts or abnormal epithelial-mesenchymal transition lead to uncontrolled myofibroblast activity and ECM deposition $(12,14)$. This may be exacerbated by cellular senescence in epithelial cells and fibroblasts, which results in increased production of profibrotic mediators (15). Senescence in type 2 alveolar epithelial cells also limits their self-renewal capacity and their ability to contribute to lung repair in the setting of epithelial injury $(16,17)$. Another proposed mechanism is dysregulated wound healing due to short telomeres. This suggestion stems from the finding that families with short telomere syndromes are predisposed to developing pulmonary fibrosis (18). Individuals with nonfamilial IPF rarely have short telomeres, however, arguing against telomere shortening as a generalized mechanism leading to IPF (19). A third proposed paradigm of IPF pathophysiology is that an inflammatory response causes dysregulated wound healing. This hypothesis has fallen out of favor in the last decade but is worth revisiting.

The inflammatory hypothesis originated from observations of a mild immune infiltrate in the lungs of individuals with IPF (although less pronounced than in individuals with other causes of fibrosis, such as connective tissue diseases) and increased levels of proinflammatory cytokines such as TNF- $\alpha$ and IL-8 in IPF lungs (20-22). Moreover, TNF- $\alpha$ was shown to be important in mouse models such as bleomycin-induced fibrosis (23). Disappointingly, a clinical trial of TNF- $\alpha$ neutralization showed no clinical benefit for individuals with IPF (24). A broader strategy for immunosuppression was attempted in the PANTHER-IPF clinical trial, where patients were treated with prednisone, azathioprine, and the antioxidant $\mathrm{N}$-acetylcysteine. Alarmingly, patients who received this experimental medication cocktail had increased rates of hospitalization and death compared with those who received placebo (25). The discouraging results from these studies were used to argue that the hypothesis of inflammation driving fibrosis in IPF was incorrect. The failure of an antiinflammatory therapy, however, does not mean that a disease is not immune mediated; it may just mean that the wrong immunomodulatory therapy was selected. For example, sepsis is due to a dysregulated host immune response to infection, but corticosteroids have not been shown to be beneficial in most studies of this disease (26). Similarly, although broadly suppressing inflammation in IPF may be harmful, this does not mean the immune system is irrelevant to the etiology or treatment of this disease.

Indeed, the importance of the immune response in IPF is suggested by genetic studies showing increased risk for or severity of disease associated with polymorphisms in the immune-related genes encoding TLR3, Toll-interacting protein (TOLLIP), and interleukin-1 receptor antagonist (IL-1RA), among others (27-30). Given these associations as well as a multitude of studies showing immune modulation of fibroblast activation and function, improved understanding of the immune system's role in IPF may enable the development of targeted immunomodulatory therapies that alter the course of the disease.

In this article, we will review the roles of various immune populations in IPF based on studies in humans and murine models.
We will primarily focus on macrophages and T cells, but will also briefly mention neutrophils, eosinophils, and B cells. We will mostly discuss studies from the last decade while referring to older literature to provide historical context. A caveat for the animal models is that they may not fully mirror IPF pathophysiology. The most widely used pulmonary fibrosis model is induced by bleomycin administration, which causes acute inflammation that progresses to fibrosis, whereas IPF is not known to have this early inflammatory phase. Bleomycin also does not induce some of the hallmark pathologic changes seen in IPF, such as lower-lobe-predominant fibrosis and clustered cystic changes known as honeycombing (31). Nevertheless, animal models provide detailed mechanistic insights that are difficult to obtain in human studies.

\section{Monocytes and macrophages}

Macrophages are one of the most abundant immune cell types in the healthylung $(32,33)$. Alveolar macrophages (AMs) play a critical role in lung homeostasis by clearing apoptotic cells and debris, regulating wound healing, and helping to initiate immune responses to lung pathogens (34). In the presence of tissue injury, monocytes are recruited into the lung and can differentiate into macrophages, further augmenting the AM pool. Macrophages are quite plastic and can adopt different functions depending on their microenvironment $(35,36)$. Historically, macrophage polarization has been understood through the M1/M2 paradigm, which we will describe below, although more recently this paradigm has been shown to be an oversimplification that belies the complexity of macrophage biology (37). In the setting of proinflammatory cytokines such as IFN- $\gamma$ or microbial products such as lipopolysaccharide (LPS), macrophages polarize into a classically activated (M1) phenotype. M1 macrophages produce proinflammatory cytokines such as IL-1 $\beta$, IL-6, and TNF- $\alpha$ as well as reactive oxygen species. These macrophages have important roles in host defense against bacterial infections. In contrast, alternatively activated (M2) macrophages develop in the presence of the Th2 cytokines IL-4 and IL-13. Subtypes of M2 macrophages can also develop in the presence of regulatory cytokines such as IL-10 or other antiinflammatory stimuli such as glucocorticoids. M2 macrophages do not produce proinflammatory cytokines and are instead characterized by expression of the macrophage mannose receptor (CD206), arginase-1, and the chitinase-like proteins $\mathrm{Ym}-1$ and $\mathrm{Ym}-2$. These macrophages are important for controlling helminth infections, regulating inflammatory (Th1) responses, and modulating white and brown adipose tissue metabolism (38-40). They can also contribute to wound healing by producing cytokines, chemokines, and growth factors that recruit and activate fibroblasts, stem/progenitor cells, and other cells involved in tissue repair (41). Lung macrophages do not neatly fit into the M1/M2 model, but the model is nevertheless helpful for understanding the role of macrophages in IPF (42).

Multiple studies of individuals with pulmonary fibrosis have shown the emergence of macrophage populations that are not present in normal lungs and may promote fibrosis. Reyfman et al. performed single-cell RNA sequencing of explanted lungs from individuals receiving transplants for IPF or other types of interstitial lung disease and compared them to biopsy samples from lung transplant donors as controls. They found that AMs from fibrotic lungs expressed higher levels of genes linked to fibrosis, such as 
IL1RN (encoding IL-1RA) and CHI3L1 (encoding chitinase-3-like protein 1). Interestingly, while AM populations from lung transplant donors were relatively homogeneous, there was substantial heterogeneity in the populations found in IPF patients (43). This is consistent with the idea that IPF is not a single disease process and that multiple pathophysiological mechanisms may lead to lung fibrosis. Allden et al. performed bronchoalveolar lavage (BAL) in individuals with IPF and healthy controls and found that BAL fluid (BALF) from IPF patients contained a population of AMs that lacked the transferrin receptor (CD71), while AMs from healthy individuals almost uniformly expressed this receptor. CD71-negative $\left(\mathrm{CD}^{-}\right)$AMs expressed high levels of the monocyte marker CD14, suggesting that they may have differentiated from circulating monocytes. Notably, the frequency of CD71- AMs correlated with subsequent mortality in these patients, suggesting that this cell population may play a detrimental role (44). In another study, BALF cells from IPF patients but not healthy controls were found to spontaneously produce high levels of CCL18, which was previously shown to be produced by M2 macrophages (45). Culture supernatants of AMs from IPF patients increased collagen production by normal lung fibroblasts in a CCL18-dependent manner. Moreover, an inverse correlation was demonstrated between CCL18 concentration in BAL-derived cell supernatants and diffusing capacity of the lungs for carbon monoxide (DLCO), a measure of lung function (46).

IPF tends to progress with a stepwise rather than continuous decline in lung function. Individuals may have stable lung function for months and then develop an event known as a flare or exacerbation with acutely worsening symptoms and an often permanent reduction in lung function (47). The cause of these flares is not well understood, and whether they are due to external stimuli such as undetected infections or result from the pathophysiology of IPF itself has been debated (48). Numerous studies have analyzed cell populations and cytokine levels in BALF from individuals with flares and observed evidence of increased inflammation, but it is unclear whether the inflammation caused the flare, or whether it is an epiphenomenon in response to another inciting event or the lung injury occurring during the flare. Nevertheless, it is worth noting that BALF cells obtained from IPF patients during flares produced higher levels of IL-1RA, CCL18, and other M2-associated chemokines compared with cells obtained from the same patients outside of flares (49). Moreover, individuals whose BALF cells produced higher amounts of CCL18 at baseline had a higher risk of future flares in this study. There have been few animal studies modeling IPF flares, but one study showed that mice with established pulmonary fibrosis infected with a gammaherpesvirus developed worsening fibrosis (50). The study's authors suggested that this occurred due to increased chemokine production leading to recruitment of circulating cells known as fibrocytes, which are monocyte-derived mesenchymal cells with properties of fibroblasts.

Murine studies have provided further mechanistic insight into the role of monocytes and M2 macrophages in pulmonary fibrosis. Misharin et al. used lineage tracing to show that in addition to the population of tissue-resident AMs (TR-AMs) that arise from fetal progenitors $(51,52)$, lung injury leads to recruitment of monocytes into the lung that differentiate into monocyte-derived AMs (Mo-AMs). These Mo-AMs persist for the lifetime of the mouse.
Through genetic deletion of caspase-8, which is necessary for differentiation of monocytes into AMs, they found that the Mo-AMs rather than TR-AMs were necessary for the development of fibrosis in response to intratracheal administration of bleomycin or an adenoviral vector encoding active TGF- $\beta$ (Ad-TGF- $\beta$ ). Notably, they also showed that corticosteroids, which had been used unsuccessfully in the PANTHER-IPF trial, did not affect Mo-AM differentiation (53). Supporting the importance of Mo-AMs, other studies showed that systemic depletion of monocytes with liposomal clodronate or prevention of monocyte trafficking to the lung through genetic deletion of CCR2 protected mice from developing lung fibrosis in response to bleomycin and Ad-TGF- $\beta(54,55)$.

How do Mo-AMs promote fibrosis? Multiple studies have implicated M2-associated genes as being responsible. Deletion of the small GTPase Rac2 in mice inhibited M2 polarization and protected from bleomycin-induced lung fibrosis (56). Injecting in vitro-polarized M2 but not M1 macrophages into Rac2-deficient mice restored sensitivity to bleomycin (57). Ucero et al. showed that mice transgenically overexpressing the AP-1 transcription factor Fra-2 have increased expression of M2 markers on their lung macrophages and spontaneously develop lung fibrosis. Conversely, Fra-2 deletion protected mice from development of bleomycin-induced fibrosis and was associated with lower M2 marker expression (58). Lung macrophages have been shown to produce several profibrotic mediators. Multiple studies have shown that TGF- $\beta$ is produced by AMs in the context of bleomycin-induced fibrosis as well as in lung sections from IPF patients $(59,60)$. Zhou et al. showed that the glycoprotein CHI3L1, which is produced by M2 macrophages, contributes to bleomycin-induced pulmonary fibrosis, possibly by promoting myofibroblast differentiation (61). Another study showed that M2 macrophages promote differentiation of lung-resident mesenchymal stem cells into myofibroblasts via the Wnt/ $\beta$-catenin pathway (62). In addition, macrophages produce multiple matrix metalloproteinases (MMPs), and several of these - including MMP3, MMP7, and MMP8 - contribute to fibrosis in the bleomycin-induced mouse model (63-65). Interestingly, in a model of vaccinia immunization that ameliorated bleomycin-induced fibrosis, vaccination was associated with an increase in M1 macrophage markers and a decrease in M2 markers in the lung (66).

Taken together, the human and mouse data suggest that monocytes are recruited into the lung in response to tissue injury and differentiate into long-lived AMs that promote fibrosis through multiple mechanisms, including production of TGF- $\beta$, CCL18, CHI3L1, MMPs, and activation of the Wnt/ $\beta$-catenin pathway that lead to fibroblast activation, myofibroblast differentiation, and ECM remodeling. Many of these profibrotic mechanisms are associated with M2 polarization, but Misharin et al. showed that Mo-AMs coexpress M1- and M2-associated markers, reinforcing the complexities of macrophage polarization in vivo (53).

What causes macrophages to produce profibrotic mediators in response to lung injury? Why do only a tiny subset of individuals develop IPF? Given the genetic associations with TLR3 and TOLLIP, it is tempting to speculate that damage-associated molecular patterns (DAMPs) produced during lung injury and pathogen-associated molecular patterns (PAMPs) produced during infections or by the lung microbiome play an important 
role. Indeed, TLR3-deficient mice displayed higher levels of the M2-promoting cytokines IL-4 and IL-13, worsened pulmonary fibrosis, and increased mortality in the bleomycin-induced fibrosis model (67). Studies of the lung microbiome in individuals with IPF have shown that they have an increased total bacterial burden compared with healthy controls and an increased prevalence of certain bacteria, including Streptococcus species $(68,69)$. Moreover, BAL bacterial burden was found to correlate with the rate of disease progression and mortality. Therefore, the lung microbiome and DAMPs produced during lung injury may predispose some individuals to develop profibrotic M2-type responses leading to pulmonary fibrosis.

Given the major role of macrophages in promoting fibrosis, future studies focused on understanding how to polarize these cells away from the production of M2-associated cytokines and chemokines toward an antifibrotic phenotype in vivo could provide promising avenues for the treatment of IPF.

\section{Neutrophils}

Kinder and colleagues studied a cohort of 156 biopsy-proven IPF patients who had bronchoscopy with BAL performed on presentation (70). They found that a subset of these individuals had elevated BAL neutrophils and reported a direct correlation between percentage of BAL neutrophils and mortality. Despite this association, it is unclear whether neutrophils themselves play a detrimental role or whether they are merely recruited in response to a pathophysiologic process that leads to mortality. Supporting a pathogenic role for neutrophils, mice deficient in neutrophil elastase were somewhat protected from asbestos-induced pulmonary fibrosis (71). On the other hand, neutrophil depletion in rats and mice did not protect against bleomycin-induced pulmonary fibrosis $(72,73)$. In other models of tissue injury, neutrophils have even been shown to protect against fibrosis (74). Therefore, the role of neutrophils in IPF pathogenesis remains equivocal.

\section{Eosinophils}

Early studies of IPF patients showed that BAL eosinophilia was relatively common and might correlate with negative outcomes (75), but these studies were performed before there was a clear diagnostic distinction between IPF and other causes of pulmonary fibrosis such as connective tissue diseases, which are more closely associated with eosinophilic responses. More recent studies have not shown a correlation between BAL eosinophilia and mortality in IPF (70). Moreover, murine studies showed no evidence for a role of eosinophils in bleomycin-induced pulmonary fibrosis, although this could be due to bleomycin being a weak stimulator of eosinophilic responses (76).

\section{T cells}

The role of adaptive immune cells in the development and progression of pulmonary fibrosis has been controversial. Numerous studies have demonstrated an increase in $\mathrm{CD} 4^{+}$and $\mathrm{CD} 8^{+} \mathrm{T}$ cells in the lungs and BAL of patients with IPF (77-79). As with the eosinophil studies discussed above, however, some of these studies were performed before IPF was clearly distinguished from other causes of pulmonary fibrosis. Moreover, it is unclear whether changes in the percentages and surface phenotypes of $\mathrm{T}$ cells in the BAL of IPF patients are functionally meaningful. As with all studies of BAL, they do not necessarily reflect changes in the lung interstitium. Studies relying on blood samples from IPF patients have shown few reproducible alterations in $\mathrm{T}$ cell numbers or phenotype, indicating how localized this disease is to the lung and the difficulty of identifying an easily measurable disease biomarker. Animal models of pulmonary fibrosis have demonstrated both pathogenic and protective roles for T cells, depending on their phenotypes. Many of these studies focused on the early inflammatory stage occurring after lung injury, however, and did not address the slow progressive fibrotic process that occurs in human disease. The animal models have shown conclusively that limiting inflammation following lung insult improves fibrosis, but this form of therapy is not applicable to IPF, which is diagnosed long after initiation of lung injury. What is clear is that the role of $\mathrm{T}$ cells in IPF is complex, with $\mathrm{T}$ cell subsets having the potential to be profibrotic, antifibrotic, or have no effect at all. Targeting $\mathrm{T}$ cells has fallen out of favor due to the failure of antiinflammatory therapies in IPF patients, but these therapies were attempted with an incomplete understanding of $\mathrm{T}$ cell subsets and the immune environment of the lung.

\section{Th1/Th2 CD4 ${ }^{+} \mathrm{T}$ cells}

Mouse models and human data have indicated that the ratio of Th1 versus Th2 $\mathrm{CD}^{+} \mathrm{T}$ cells in the lung may dictate the course of lung injury and determine whether there is resolution or progression to fibrosis (80-82). Increased concentrations of IL-4 and IL-13 and decreased concentrations of IFN- $\gamma$ have been observed in BAL of IPF patients as well as in mouse models (83-85). IL-13 was shown to induce proliferation of lung fibroblasts and differentiation of monocytes into collagen-expressing fibrocytes (86, 87). IL-4 and IL-13 also induce M2 macrophage polarization. In contrast, IL-12- and IFN- $\gamma$-producing Th1 cells inhibit fibrocyte differentiation and promote generation of M1 macrophages (87, 88). Based on these data, a therapy that promotes differentiation of Th1 over Th2 cells would seem promising. Unfortunately, clinical trials that administered recombinant IFN- $\gamma$ or an anti-IL-13 monoclonal antibody failed to show any clinical benefit in IPF patients $(89,90)$. These failures likely reflect the fact that $\mathrm{T}$ cell differentiation does not simply result in the generation of a single cytokine, but rather an entire metabolic, epigenetic, and phenotypic reprogramming of cell function. Decreased IFN- $\gamma$-producing Th1 cells in the lungs of IPF patients do not represent a defect in IFN- $\gamma$ production, but rather an altered lung environment that skews T cells to alternate differentiation pathways, such as Th17 or regulatory $\mathrm{T}$ cells (Tregs). This immune dysregulation in turn permits abnormal wound healing. Addition of exogenous IFN- $\gamma$ or anti-IL-13 alone cannot cause the redifferentiation of lung T cells into Th1 cells. To fully leverage the antifibrotic function of Th1 cells in IPF, a more immunologically relevant way to promote $\mathrm{T}$ cell differentiation in vivo would be needed.

\section{Th17 CD4 ${ }^{+} \mathrm{T}$ cells}

There are multiple studies regarding the role of Th17 cells in fibrosis. The consensus is that they are profibrotic and therefore detrimental in IPF. Supporting this is the observation that concentrations of IL-17A and IL-1 $\beta$ are elevated in the BALF of IPF 

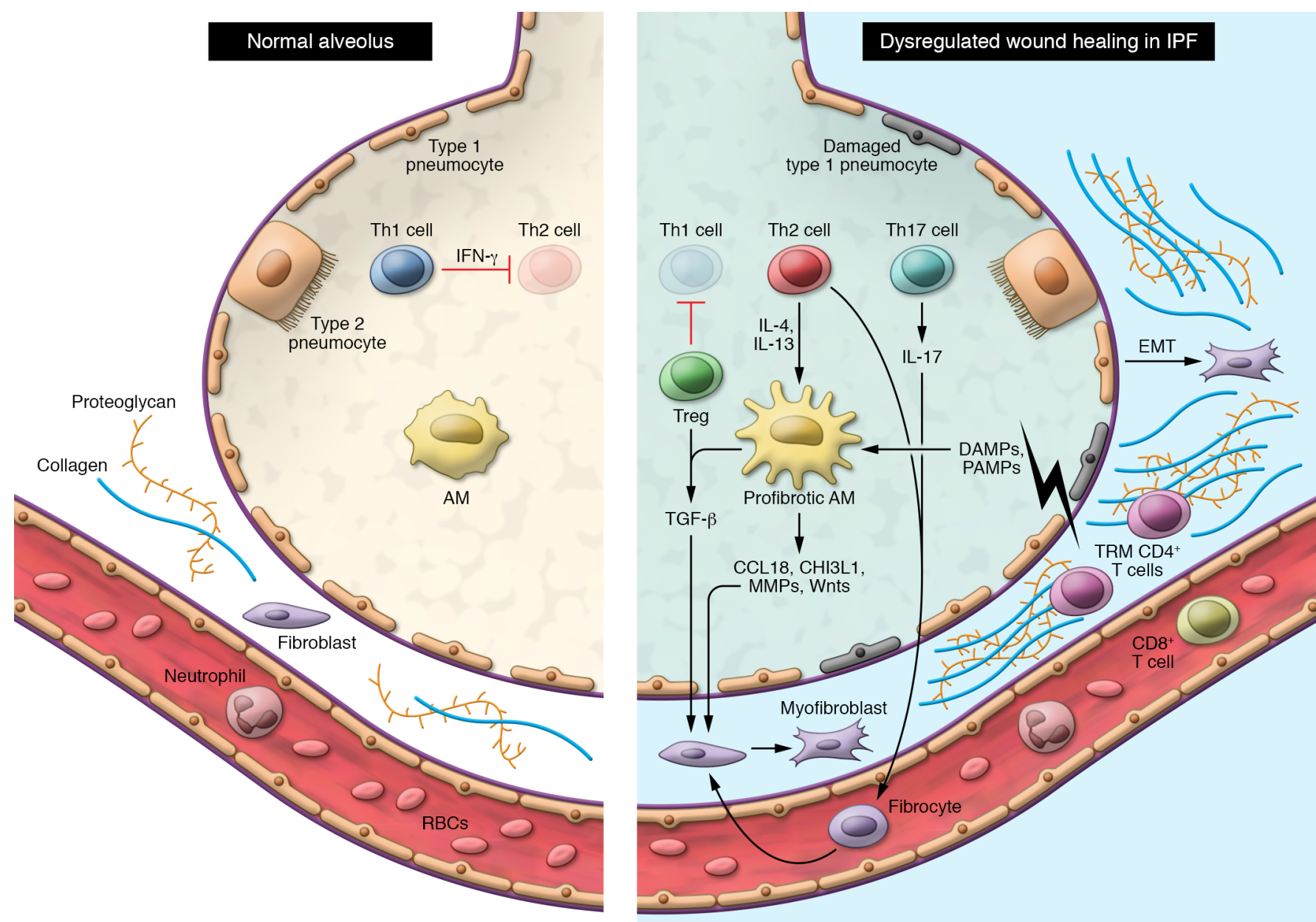

Lung capillary

Figure 1. In individuals with idiopathic pulmonary fibrosis, lung epithelial injury leads to production of profibrotic cytokines and chemolkines such as CCL18, CHI3L1, MMPs, Wnt, and TGF- $\beta$ by alveolar macrophages. This results in activation of fibroblasts and differentiation into myofibroblasts, which produce extracellular matrix leading to thickening of the lung interstitium. Abnormal epithelial-mesenchymal transition (EMT) also contributes to the pool of myofibroblasts in the lung interstitium. Regulatory T cells inhibit Th1 cell activation, resulting in an altered Th1/Th2 balance in the lung. Production of IL-4 and IL-13 by Th2 cells promotes polarization of alveolar macrophages into an M2 profibrotic phenotype and differentiation of circulating fibrocytes into fibroblasts. IL-17 production by Th17 cells in the lung also promotes fibroblast activation. CD8 ${ }^{+} \mathrm{T}$ cells and TRM CD4 ${ }^{+} \mathrm{T}$ cells appear to protect against fibrosis, although the mechanisms through which they do so are not well understood.

patients (91). Moreover, lung fibroblasts from IPF patients express the IL-17 receptor and respond to IL-17 by producing ECM and proliferating (92). Mouse models of bleomycin- or radiation-induced fibrosis demonstrate increased lung $\mathrm{CD} 4^{+} \mathrm{IL}-17^{+} \mathrm{T}$ cells and IL-17-deficient mice have decreased pulmonary fibrosis $(93,94)$. Alterations in lung commensal bacteria have also been reported to promote pulmonary fibrosis through induction of Th17 cells (95), which may help explain the lung microbiome changes observed in individuals with IPF. Recently, it was reported that IPF patients have increased expression of the checkpoint inhibitor PD-1 on $\mathrm{CD} 4^{+} \mathrm{T}$ cells in their blood and in pulmonary biopsies. Interestingly, the majority of these PD- $1^{+}$T cells expressed IL-17 and TGF- $\beta$ and failed to proliferate upon stimulation. PD-1 pathway blockade decreased IL-17 and TGF- $\beta$ production by these cells (96). These data suggest that PD-1 blockade might redirect $\mathrm{T}$ cells away from a profibrotic Th17 response. Arguing against this therapeutic approach, however, are recent reports indicating that anti-PD-1 immunotherapy can cause interstitial lung disease through an unclear mechanism (97).

\section{Tregs}

There are conflicting data regarding the role of Tregs in IPF. Initially, it was reported that IPF patients had decreased numbers of Tregs in blood and BALF and that their Tregs were less suppressive than those from healthy controls (98). Subsequent studies, however, reported increased numbers of Tregs in the blood and BALF of IPF patients and increased TGF- $\beta$ production by circulating Tregs upon stimulation (99-101). In several different mouse models of pulmonary fibrosis, depletion of Tregs resulted in attenuated fibrosis, while induction or transfer of Tregs resulted in worsened fibrosis (102-104). Several of these reports suggested this was due to skewing of lung $\mathrm{CD}^{+} \mathrm{T}$ cells away from $\mathrm{Th} 1$ 
differentiation $(102,104)$. In the bleomycin-induced pulmonary fibrosis model, Treg depletion was also associated with a decrease in lung Th17 cells (103). These findings indicate that IPF may be immunologically more similar to cancer than to an inflammatory or autoimmune disease, with disease progression being due to a dysregulated immune response rather than an overexuberant one. Nevertheless, while the data on Tregs in animal models support their profibrotic role, more studies are needed to clarify their involvement in the progression of human IPF.

\section{CD8 ${ }^{+} \mathrm{T}$ cells}

In fibrotic human lungs, $\mathrm{CD}^{+} \mathrm{T}$ cells tend to aggregate near lymphoid follicles, while $\mathrm{CD} 8^{+} \mathrm{T}$ cells are often found more diffusely throughout the lung parenchyma and alveolar walls (105). This spatial separation alludes to these cells having different roles in the course of this disease. Increased $\mathrm{CD}^{+} \mathrm{T}$ cells in lung biopsies of IPF patients were found to correlate with decreased total lung capacity and forced vital capacity (105). Increased numbers of CD28-negative CD8 ${ }^{+} \mathrm{T}$ cells have also been observed in IPF patient lung explants. These cells have a profibrotic and proinflammatory transcriptional profile and express increased levels of PD-1, which together with the loss of CD28 suggests a state of chronic activation (106). In the bleomycin-induced pulmonary fibrosis model, $\mathrm{CD}^{+} \mathrm{T}$ cells were shown to differentiate into profibrotic IL-13-producing cells through ligation of the IL-21 receptor. Moreover, treating these cells with IL- 4 and IL-21 caused them to produce additional IL-21, promoting IL-13 production in an autocrine manner (107). Overall, the data on $\mathrm{CD}^{+} \mathrm{T}$ cells point to their detrimental role in the development and progression of pulmonary fibrosis, indicating a potential therapeutic target.

\section{Tissue-resident memory cells}

Recently, there has been interest in the role of tissue-resident memory (TRM) cells in disease. The available data on TRM cells in pulmonary fibrosis are limited, but several important findings have been made. The first was the observation that BALF from IPF patients contains an increased number of TRM CD4 ${ }^{+} \mathrm{T}$ cells expressing the integrin CD103 (108). In addition, improved DLCO and survival were observed in IPF patients who had an increased number of circulating resting memory $\mathrm{CD} 4^{+} \mathrm{T}$ cells (109). In a mouse model of bleomycin-induced chronic fibrosis, a vaccination that induced an increase in tissue-resident $\mathrm{CD} 4^{+} \mathrm{T}$ cells in the lung was effective at reducing established fibrosis and improved pulmonary function (110). These data indicate a potential beneficial role for TRM cells and a possibility for tissue-specific therapy, although more studies are necessary to fully understand these cells' complex functions. In contrast to these data, a recent report identified a population of $\mathrm{CD} 8^{+} \mathrm{TRM}$ cells that displayed an exhausted phenotype following influenza infection. Rejuvenation of these exhausted TRM cells with anti-PD1 therapy resulted in increased lung hydroxyproline concentrations (111). While these experiments were not performed in a traditional fibrosing model, they indicate a potentially pathogenic role for $\mathrm{CD}^{+} \mathrm{TRM}$ cells.

Taken together, studies examining the role of T cells in IPF suggest that targeting a single $\mathrm{T}$ cell-derived cytokine is unlikely to be sufficient to treat the disease. Rather, future studies should focus on in vivo strategies for modifying $\mathrm{T}$ cell differentiation in the lung to promote Th1 and TRM CD4 ${ }^{+} \mathrm{T}$ cell responses, while suppressing Th2 and Th17 responses after tissue injury.

\section{B cells}

Similarly to T cells, B cells are increased in the lungs of IPF patients. Phenotypically, circulating B cells from IPF patients have been shown to be more antigen-differentiated, have a higher proportion of plasmablasts, and express fewer regulatory markers. Additionally, serum levels of B lymphocyte stimulating factor (BLyS) were significantly higher in IPF patients and correlated with decreased survival (112). In the bleomycin model, loss of CD19 attenuated inflammation and reduced mortality, while CD19 overexpression increased mortality (113). Consistent with increased B cell activation, circulating IgG and IgA antibodies are increased in IPF patients $(114,115)$. Surprisingly, an increased percentage of these antibodies appear to be autoreactive, with autoreactive serum IgA levels correlating with a decrease in forced vital capacity in one study (115). Conversely, however, a recent report showed antibodies against Hsp72 in BALF of IPF patients, with higher concentrations correlating with improved survival (114). The mechanism of this beneficial effect is unclear, but the authors of the study postulated that the antibodies modulate macrophage activation. Overall, these data indicate that the quantity and phenotype of B cells are altered in IPF, leading to an increase in serum and lung antibodies, some of which are autoreactive. Whether activated B cells and autoantibodies induce lung fibrosis or are induced as a result of inflammation which then drives fibrotic progression is unclear.

\section{TGF- $\beta$}

Although it does not fit neatly into the sections above, any review of fibrosis is incomplete without a discussion of TGF- $\beta$. This cytokine is considered central to the pathophysiology of fibrosis by promoting fibroblast proliferation, myofibroblast differentiation, ECM production, and epithelial-mesenchymal transition (116). Overexpression of TGF- $\beta$ in the lung is sufficient to cause pulmonary fibrosis (117) and mice with impaired TGF- $\beta$ signaling due to Smad3 deletion are resistant to bleomycin-induced pulmonary fibrosis (118).

There are three mammalian isoforms of TGF- $\beta$, each encoded by a different gene. TGF- $\beta 1$ is the most abundantly and ubiquitously expressed and has been extensively studied in pulmonary fibrosis, but TGF- $\beta 2$ and TGF- $\beta 3$ signal through the same receptors and may also have profibrotic roles (119). TGF- $\beta 1$ is produced by multiple cell types, including epithelial cells, fibroblasts/myofibroblasts, macrophages, and Tregs. It is initially synthesized in association with a latency-associated peptide (LAP) and latent TGF- $\beta$-binding protein (LTBP), which form a complex that sequesters TGF- $\beta 1$ from its receptors. The latent TGF- $\beta 1$ complex binds to ECM, forming a reservoir of TGF- $\beta 1$ that can be rapidly activated. During lung injury or inflammation, proteases such as MMPs, plasmin, and thrombin cleave the complex and proteins such as $\alpha \mathrm{V} \beta 6$ and $\alpha \mathrm{V} \beta 8$ integrins bind to LAP and induce a conformational change that liberates bioactive TGF- $\beta 1$ (120).

Given the central role of TGF- $\beta$ in fibrosis, directly targeting this molecule might seem to be an obvious treatment approach, but this is not a simple matter, as TGF- $\beta$ is critical in immune 
regulation. Indeed, deletion of the $T g f b 1$ gene leads to fatal multiorgan autoimmunity in mice (121). A TGF- $\beta 1$-specific neutralizing antibody showed no efficacy in treating scleroderma (122), so an antibody that neutralizes all three isoforms of TGF- $\beta$ was developed. This antibody was tested in a small open-label trial of scleroderma patients and did show some evidence of efficacy (123), but no follow-up trials have been published. A phase I trial of this antibody performed in IPF patients (NCT00125385) remains unpublished. An alternative approach was attempted in a phase II trial using an anti- $\alpha \mathrm{V} \beta 6$ antibody to prevent TGF- $\beta$ activation, but the trial was halted due to safety concerns (NCT03573505). It therefore remains uncertain whether TGF- $\beta$ can be safely targeted for the treatment of IPF. Future studies examining lung-specific inhibition of TGF- $\beta$ (for example through nebulized delivery of therapeutic agents) would be helpful given the pleiotropic effects of this cytokine and potential adverse effects of inhibiting it systemically.

\section{Conclusion}

Studies in individuals with IPF and mouse models strongly support a role for immune dysregulation in promoting the develop- ment of pulmonary fibrosis. M2 macrophages, Th17 cells, CD8 ${ }^{+}$ $\mathrm{T}$ cells, and possibly Tregs promote fibrosis, while Th1 and TRM $\mathrm{CD}^{+} \mathrm{T}$ cells appear to be protective (Figure 1). Although early trials targeting inflammation in IPF were unsuccessful, a more nuanced understanding of the role of the immune system in IPF may lead to the development of targeted immune modulators that are efficacious in this disease. For example, therapies that skew macrophage polarization toward an antifibrotic phenotype, promote $\mathrm{CD} 4^{+} \mathrm{T}$ cell differentiation into Th1 or TRM cells, or inhibit lung-specific TGF- $\beta$ activation by modulating the local inflammatory milieu might be beneficial. Much as immune checkpoint blockade and cellular therapies (e.g., CAR-T cells) have dramatically changed the cancer treatment landscape, such approaches hold great promise in potentially inhibiting and reversing the dysregulated processes leading to unremitting fibrosis in IPF.

Address correspondence to: Maureen R. Horton, Department of Medicine, Division of Pulmonary and Critical Care, Johns Hopkins University School of Medicine, 1830 E. Monument St., 5th Floor, Baltimore, Maryland 21205, USA. Phone: 410.955.4176; Eamil:mhorton2@jhmi.edu.
1. Raghu G, et al. Incidence and prevalence of idiopathic pulmonary fibrosis in US adults 18-64 years old. Eur Respir J. 2016;48(1):179-186.

2. Ley B, Collard HR. Epidemiology of idiopathic pulmonary fibrosis. Clin Epidemiol. 2013;5(1):483-492.

3. Nalysnyk L, et al. Incidence and prevalence of idiopathic pulmonary fibrosis: review of the literature. Eur Respir Rev. 2012;21(126):355-361.

4. King TE, et al. A phase 3 trial of pirfenidone in patients with idiopathic pulmonary fibrosis. N Engl J Med. 2014;370(22):2083-2092.

5. Richeldi L, et al. Efficacy and safety of nintedanib in idiopathic pulmonary fibrosis. $\mathrm{NEnglJM}$ Med. 2014;370(22):2071-2082.

6. Raghu G, et al. An official ATS/ERS/JRS/ALAT clinical practice guideline: treatment of idiopathic pulmonary fibrosis: An update of the 2011 clinical practice guideline. Am J Respir Crit Care Med. 2015;192(2):e3-e19.

7. Raghu G, et al. Idiopathic pulmonary fibrosis in US Medicare beneficiaries aged 65 years and older: Incidence, prevalence, and survival, 2001-11. Lancet Respir Med. 2014;2(7):566-572.

8. [no authors listed]. American Thoracic Society. Idiopathic pulmonary fibrosis: diagnosis and treatment. Am J Respir Crit Care Med. 2000;161(2 pt 1):646-664.

9. [no authors listed]. American Thoracic Society/ European Respiratory Society international multidisciplinary consensus classification of the idiopathic interstitial pneumonias. This joint statement of the American Thoracic Society (ATS), and the European Respiratory Society (ERS) was adopted by the ATS board of directors, June 2001 and by the ERS Executive Committee, June 2001. Am J Respir Crit Care Med. 2002;(2):277-304.

10. King TE, et al. Predicting survival in idiopathic pulmonary fibrosis: Scoring system and survival model. Am J Respir Crit Care Med. 2001;164(7):1171-1181.
11. Betensley A, et al. A Systematic review of the role of dysfunctional wound healing in the pathogenesis and treatment of idiopathic pulmonary fibrosis. JClin Med. 2016;6(1):2.

12. Selman M, et al. Idiopathic pulmonary fibrosis: prevailing and evolving hypotheses about its pathogenesis and implications for therapy. Ann Intern Med. 2001;134(2):136-151.

13. Stewart JP, et al. The detection of Epstein-Barr virus DNA in lung tissue from patients with idiopathic pulmonary fibrosis. Am J Respir Crit Care Med.1999;159(4 pt 1):1336-1341.

14. Noble PW. Epithelial fibroblast triggering and interactions in pulmonary fibrosis. Eur Respir Rev. 2008;17(109):123-129.

15. Schafer MJ, et al. Cellular senescence mediates fibrotic pulmonary disease. Nat Commun. 2017;8(1):14532.

16. Parimon T, et al. Alveolar epithelial type II cells as drivers of lung fibrosis in idiopathic pulmonary fibrosis. Int J Mol Sci. 2020;21(7):2269.

17. Liang J, et al. Hyaluronan and TLR4 promote surfactant-protein-C-positive alveolar progenitor cell renewal and prevent severe pulmonary fibrosis in mice. Nat Med. 2016;22(11):1285-1293.

18. Armanios MY, et al. Telomerase mutations in families with idiopathic pulmonary fibrosis. N Engl J Med. 2007;356(13):1317-1326.

19. Liu T, et al. Telomerase and telomere length in pulmonary fibrosis. Am J Respir Cell Mol Biol. 2013;49(2):260-268.

20. Parra ER, et al. Inflammatory cell phenotyping of the pulmonary interstitium in idiopathic interstitial pneumonia. Respiration. 2007;74(2):159-169.

21. Kapanci Y, et al. Cytoskeletal protein modulation in pulmonary alveolar myofibroblasts during idiopathic pulmonary fibrosis: Possible role of transforming growth factor beta and tumor necrosis factor alpha. Am J Respir Crit Care Med. 1995;152(6 pt 1):2163-2169.

22. Carre PC, et al. Increased expression of the interleukin-8 gene by alveolar macrophages in idiopathic pulmonary fibrosis. A potential mechanism for the recruitment and activation of neutrophils in lung fibrosis. J Clin Invest. 1991;88(6):1802-1810.

23. Ortiz LA, et al. Expression of the TNF and the necessity of TNF receptors in bleomycininduced lung injury in mice. Exp Lung Res. 1998;24(6):721-743.

24. Raghu G, et al. Treatment of idiopathic pulmonary fibrosis with etanercept: An exploratory, placebo-controlled trial. Am J Respir Crit Care Med. 2008;178(9):948-955.

25. Raghu G, et al. Prednisone, azathioprine, and $\mathrm{N}$-acetylcysteine for pulmonary fibrosis. $\mathrm{N} \mathrm{EnglJ}$ Med. 2012;366(21):1968-1977.

26. Sprung CL, et al. Hydrocortisone therapy for patients with septic shock. N EnglJMed. 2008;358(2):111-124.

27. O'Dwyer DN, et al. The Toll-like receptor $3 \mathrm{~L} 412 \mathrm{~F}$ polymorphism and disease progression in idiopathic pulmonary fibrosis. Am J Respir Crit Care Med. 2013;188(12):1442-1450.

28. Noth I, et al. Genetic variants associated with idiopathic pulmonary fibrosis susceptibility and mortality: a genome-wide association study. Lancet Respir Med. 2013;1(4):309-317.

29 . Whyte $M$, et al. Increased risk of fibrosing alveolitis associated with interleukin-1 receptor antagonist and tumor necrosis factor- $\alpha$ gene polymorphisms. Am J Respir Crit Care Med. 2000;162(2 pt 1):755-758.

30. Korthagen NM, et al. IL1RN genetic variations and risk of IPF: a meta-analysis and mRNA expression study. Immunogenetics. 2012;64(5):371-377.

31. Moore BB, et al. Animal models of fibrotic lung disease. Am J Respir Cell Mol Biol. 2013;49(2):167-179.

32. Desch AN, et al. Flow cytometric analysis of mononuclear phagocytes in nondiseased human lung and lung-draining lymph nodes. Am J Respir 
Crit Care Med. 2016;193(6):614-626.

33. Yu Y-RA, et al. A protocol for the comprehensive flow cytometric analysis of immune cells in normal and inflamed murine non-lymphoid tissues. PLoS One. 2016;11(3):e0150606.

34. Hussell T, Bell TJ. Alveolar macrophages: plasticity in a tissue-specific context. Nat Rev Immunol. 2014;14(2):81-93.

35. Murray PJ. Macrophage polarization. Annu Rev Physiol. 2017;79(1):541-566.

36. Mills CD, et al. M-1/M-2 macrophages and the Th1/Th2 paradigm. JImmunol. 2000;164(12):6166-6173.

37. Murray PJ, et al. Macrophage activation and polarization: nomenclature and experimental guidelines. Immunity. 2014;41(1):14-20.

38. Anthony RM, et al. Memory TH2 cells induce alternatively activated macrophages to mediate protection against nematode parasites. Nat Med. 2006;12(8):955-960.

39. Herbert DR, et al. Alternative macrophage activation is essential for survival during schistosomiasis and downmodulates T helper 1 responses and immunopathology. Immunity. 2004;20(5):623-635.

40. Hallowell RW, et al. mTORC2 signalling regulates M2 macrophage differentiation in response to helminth infection and adaptive thermogenesis. Nat Commun. 2017;8(1):14208.

41. Wynn TA, Vannella KM. Macrophages in tissue repair, regeneration, and fibrosis. Immunity. 2016;44(3):450-462.

42. Mitsi E, et al. Human alveolar macrophages predominately express combined classical M1 and M2 surface markers in steady state. Respir Res. 2018;19(1):66.

43. Reyfman PA, et al. Single-cell transcriptomic analysis of human lung provides insights into the pathobiology of pulmonary fibrosis. Am J Respir Crit Care Med. 2019;199(12):1517-1536.

44. Allden SJ, et al. The transferrin receptor CD71 delineates functionally distinct airway macrophage subsets during idiopathic pulmonary fibrosis. Am J Respir Crit Care Med. 2019;200(2):209-219.

45. Kodelja V, et al. Alternative macrophage activation-associated CC-chemokine-1, a novel structural homologue of macrophage inflammatory protein-1 alpha with a Th2-associated expression pattern. JImmunol. 1998;160(3):1411-1418.

46. Prasse A, et al. A vicious circle of alveolar macrophages and fibroblasts perpetuates pulmonary fibrosis via CCL18. Am J Respir Crit Care Med. 2006;173(7):781-792.

47. Ley B, et al. Clinical course and prediction of survival in idiopathic pulmonary fibrosis. Am J Respir Crit Care Med. 2011;183(4):431-440.

48. Collard HR, et al. Acute exacerbation of idiopathic pulmonary fibrosis an international working group report. Am J Respir Crit Care Med. 2016;194(3):265-275.

49. Schupp JC, et al. Macrophage activation in acute exacerbation of idiopathic pulmonary fibrosis. PLoS One. 2015;10(1):e0116775.

50. McMillan TR, et al. Exacerbation of established pulmonary fibrosis in a murine model by gammaherpesvirus. Am J Respir Crit Care Med. 2008;177(7):771-780.

51. Guilliams M, et al. Alveolar macrophages develop from fetal monocytes that differentiate into longlived cells in the first week of life via GM-CSF. J Exp Med. 2013;210(10):1977-1992.

52. Sheng J, et al. Most tissue-resident macrophages except microglia are derived from fetal hematopoietic stem cells. Immunity. 2015;43(2):382-393.

53. Misharin AV., et al. Monocyte-derived alveolar macrophages drive lung fibrosis and persist in the lung over the life span. J Exp Med. 2017;214(8):2387-2404.

54. Moore BB, et al. Protection from pulmonary fibrosis in the absence of CCR2 signaling. J Immunol. 2001;167(8):4368-4377.

55. Gibbons MA, et al. Ly6 $\mathrm{C}^{\text {hi }}$ monocytes direct alternatively activated profibrotic macrophage regulation of lung fibrosis. Am J Respir Crit Care Med. 2011;184(5):569-581.

56. Arizmendi N, et al. Rac 2 is involved in bleomycin-induced lung inflammation leading to pulmonary fibrosis. Respir Res. 2014;15(1):71.

57. Joshi $\mathrm{S}$, et al. Rac2 is required for alternative macrophage activation and bleomycin induced pulmonary fibrosis; a macrophage autonomous phenotype. PLoS One. 2017;12(8):e0182851.

58. Ucero AC, et al. Fra-2-expressing macrophages promote lung fibrosis. J Clin Invest. 2019;129(8):3293-3309.

59. Khalil N, et al. Macrophage production of transforming growth factor $\beta$ and fibroblast collagen synthesis in chronic pulmonary inflammation. JExp Med.1989;170(3):727-737.

60. Khalil N, et al. Increased production and immunohistochemical localization of transforming growth factor-beta in idiopathic pulmonary fibrosis. Am J Respir Cell Mol Biol. 1991;5(2):155-162.

61. Zhou Y, et al. Chitinase 3-like 1 suppresses injury and promotes fibroproliferative responses in mammalian lung fibrosis. Sci Transl Med. 2014;6(240):240ra76.

62. Hou J, et al. M2 macrophages promote myofibroblast differentiation of LR-MSCs and are associated with pulmonary fibrogenesis. Cell Commun Signal. 2018;16(1):89.

63. Yamashita CM, et al. Matrix metalloproteinase 3 is a mediator of pulmonary fibrosis. Am J Pathol. 2011;179(4):1733-1745.

64. Zuo F, et al. Gene expression analysis reveals matrilysin as a key regulator of pulmonary fibrosis in mice and humans. Proc Natl Acad Sci U S A. 2002;99(9):6292-6297.

65. García-Prieto E, et al. Resistance to bleomycininduced lung fibrosis in MMP-8 deficient mice is mediated by interleukin-10. PLoS One. 2010;5(10):e13242.

66. Collins SL, et al. Pulmonary vaccination as a novel treatment for lung fibrosis. PLoS One. 2012;7(2):e31299.

67. O'Dwyer DN, et al. Lung microbiota contribute to pulmonary inflammation and disease progression in pulmonary fibrosis. Am J Respir Crit Care Med. 2019;199(9):1127-1138.

68. Molyneaux PL, et al. The role of bacteria in the pathogenesis and progression of idiopathic pulmonary fibrosis. Am J Respir Crit Care Med. 2014;190(8):906-913.

69. Han MLK, et al. Lung microbiome and disease progression in idiopathic pulmonary fibrosis: an analysis of the COMET study. Lancet Respir Med.
2014;2(7):548-556

70. Kinder BW, et al. Baseline BAL neutrophilia predicts early mortality in idiopathic pulmonary fibrosis. Chest. 2008;133(1):226-232.

71. Gregory AD, et al. Neutrophil elastase promotes myofibroblast differentiation in lung fibrosis. JLeukoc Biol. 2015;98(2):143-152.

72. Thrall RS, et al. The development of bleomycininduced pulmonary fibrosis in neutrophildepleted and complement-depleted rats. Am J Pathol.1981;105(1):76-81.

73. Manoury B, et al. Influence of early neutrophil depletion on MMPs/TIMP-1 balance in bleomycin-induced lung fibrosis. Int Immunopharmacol. 2007;7(7):900-911.

74. Calvente CJ, et al. Neutrophils contribute to spontaneous resolution of liver inflammation and fibrosis via microRNA-223. J Clin Invest. 2019;129(10):4091-4109.

75. Boomars KA, et al. Relationship between cells obtained by bronchoalveolar lavage and survival in idiopathic pulmonary fibrosis. Thorax. 1995;50(10):1087-1092.

76. Hao H, et al. Bleomycin-induced pulmonary fibrosis is independent of eosinophils. J Leukoc Biol. 2000;68(4):515-521.

77. Papiris SA, et al. Relationship of BAL and lung tissue $\mathrm{CD}^{+}$and $\mathrm{CD}^{+} \mathrm{T}$ lymphocytes, and their ratio in idiopathic pulmonary fibrosis. Chest. 2005;128(4):2971-2977.

78. Nuovo GJ, et al. The distribution of immunomodulatory cells in the lungs of patients with idiopathic pulmonary fibrosis. Mod Pathol. 2012;25(3):416-433.

79. Herazo-Maya JD, et al. Peripheral blood mononuclear cell gene expression profiles predict poor outcome in idiopathic pulmonary fibrosis. Sci Transl Med. 2013;5(205):205ra136.

80. Hoffmann KF, et al. Disease fingerprinting with cDNA microarrays reveals distinct gene expression profiles in lethal type 1 and type 2 cytokine-mediated inflammatory reactions. FASEB J. 2001;15(13):2545-2547.

81. Sandler NG, et al. Global gene expression profiles during acute pathogen-induced pulmonary inflammation reveal divergent roles for Th1 and Th2 responses in tissue repair. J Immunol. 2003;171(7):3655-3667.

82. Hesse $M$, et al. Differential regulation of nitric oxide synthase- 2 and arginase- 1 by Type 1 /Type 2 cytokines in vivo: granulomatous pathology is shaped by the pattern of l-arginine metabolism. JImmunol. 2001;167(11):6533-6544.

83. Emura M, et al. In vitro production of B cell growth factor and B cell differentiation factor by peripheral blood mononuclear cells and bronchoalveolar lavage T lymphocytes from patients with idiopathic pulmonary fibrosis. Clin Exp Immunol. 2008;82(1):133-139.

84. Zhu Z, et al. Pulmonary expression of interleukin-13 causes inflammation, mucus hypersecretion, subepithelial fibrosis, physiologic abnormalities, and eotaxin production. JClin Invest. 1999;103(6):779-788.

85. Belperio JA, et al. Interaction of IL-13 and C10 in the pathogenesis of bleomycin-induced pulmonary fibrosis. Am J Respir Cell Mol Biol. 2002;27(4):419-427. 
86. Saito A, et al. Potential action of IL-4 and IL-13 as fibrogenic factors on lung fibroblasts in vitro. Int Arch Allergy Immunol. 2003;132(2):168-176.

87. Shao DD, et al. Pivotal advance: Th-1 cytokines inhibit, and Th-2 cytokines promote fibrocyte differentiation. JLeukoc Biol. 2008;83(6):1323-1333.

88. Gordon S. Alternative activation of macrophages. Nat Rev Immunol. 2003;3(1):23-35.

89. King TE, et al. Effect of interferon gamma-1b on survival in patients with idiopathic pulmonary fibrosis (INSPIRE): a multicentre, randomised, placebo-controlled trial. Lancet. 2009;374(9685):222-228.

90. Parker JM, et al. A phase 2 randomized controlled study of tralokinumab in subjects with idiopathic pulmonary fibrosis. Am J Respir Crit Care Med. 2018;197(1):94-103.

91. Wilson MS, et al. Bleomycin and IL-1 $\beta$-mediated pulmonary fibrosis is IL-17A dependent. JExp Med. 2010;207(3):535-552.

92. Zhang J, et al. Profibrotic effect of IL-17A and elevated IL-17RA in idiopathic pulmonary fibrosis and rheumatoid arthritis-associated lung disease support a direct role for IL-17A/IL-17RA in human fibrotic interstitial lung disease. Am J Physiol Cell Mol Physiol. 2019;316(3):L487-L497.

93. Cipolla E, et al. IL-17A deficiency mitigates bleomycin-induced complement activation during lung fibrosis. FASEB J. 2017;31(12):5543-5556.

94. Paun A, et al. The Th1/Th17 balance dictates the fibrosis response in murine radiation-induced lung disease. Sci Rep. 2017;7(1):11586.

95. Yang D, et al. Dysregulated lung commensal bacteria drive interleukin-17B production to promote pulmonary fibrosis through their outer membrane vesicles. Immunity. 2019;50(3):692-706.

96. Celada LJ, et al. PD-1 up-regulation on $\mathrm{CD}^{+}$ $\mathrm{T}$ cells promotes pulmonary fibrosis through STAT3-mediated IL-17A and TGF- $\beta 1$ production. Sci Transl Med. 2018;10(460):eaar8356.

97. Akella P, et al. Anti PD-1 immunotherapy related interstitial lung disease presenting as respiratory failure - a review with case series. Respir Med Case Rep. 2019;26:17-22.

98. Kotsianidis I, et al. Global impairment of $\mathrm{CD}^{+}$ $\mathrm{CD}^{2} 5^{+} \mathrm{FOXP}^{+}$regulatory $\mathrm{T}$ cells in idiopathic pulmonary fibrosis. Am J Respir Crit Care Med. 2009;179(12):1121-1130.

99. Galati D, et al. Peripheral depletion of NK cells and imbalance of the Treg/Th17 axis in idiopathic pulmonary fibrosis patients. Cytokine. 2014;66(2):119-126.

100. Hou Z, et al. Increased activated regulatory $\mathrm{T}$ cells proportion correlate with the severity of idiopathic pulmonary fibrosis. Respir Res. 2017;18(1):170.

101. Milger K, et al. Pulmonary CCR $2^{+} \mathrm{CD} 4^{+} \mathrm{T}$ cells are immune regulatory and attenuate lung fibrosis development. Thorax. 2017;72(11):1007-1020.

102. Birjandi SZ, et al. CD $4^{+} C D 25^{\text {hi }}$ Foxp $3^{+}$cells exacerbate bleomycin-induced pulmonary fibrosis. Am J Pathol. 2016;186(8):2008-2020.

103. Chakraborty K, et al. Impact of Treg on other $\mathrm{T}$ cell subsets in progression of fibrosis in experimental lung fibrosis. Tissue Cell. 2018;53:87-92.

104. Xiong S, et al. Treg depletion attenuates irradiation-induced pulmonary fibrosis by reducing fibrocyte accumulation, inducing Th17 response, and shifting IFN- $\gamma$, IL-12/IL-4, IL-5 balance. Immunobiology. 2015;220(11):1284-1291.

105. Daniil Z, et al. CD8 ${ }^{+} \mathrm{T}$ lymphocytes in lung tissue from patients with idiopathic pulmonary fibrosis Respir Res. 2005;6(1):81.

106. Habiel DM, et al. Characterization of CD28 null T cells in idiopathic pulmonary fibrosis. Mucosal Immunol. 2019;12(1):212-222.

107. Brodeur TY, et al. IL-21 promotes pulmonary fibrosis through the induction of profibrotic CD8 T cells. J Immunol. 2015;195(11):5251-5260.

108. Braun RK, et al. Phenotypic and molecular characterization of $\mathrm{CD} 103^{+} \mathrm{CD} 4^{+} \mathrm{T}$ cells in bronchoalveolar lavage from patients with interstitial lung diseases. Cytometry B Clin Cytom. 2003;54(1):19-27.

109. Liu YZ, et al. Proportions of resting memory $\mathrm{T}$ cells and monocytes in blood have prognostic significance in idiopathic pulmonary fibrosis. Genomics. 2019;111(6):1343-1350.

110. Collins SL, et al. Vaccinia vaccine-based immunotherapy arrests and reverses established pulmonary fibrosis. JCI Insight. 2016;1(4):e83116.

111. Wang $\mathrm{Z}$, et al. PD- $1^{\text {hi }} \mathrm{CD} 8^{+}$resident memory $\mathrm{T}$ cells balance immunity and fibrotic sequelae. $\mathrm{Sci}$ Immunol. 2019;4(36):eaaw1217.

112. Xue J, et al. Plasma B lymphocyte stimulator and B cell differentiation in idiopathic pulmonary fibrosis patients. J Immunol. 2013;191(5):2089-2095.

113. Komura K, et al. CD19 regulates the development of bleomycin-induced pulmonary fibrosis in a mouse model. Arthritis Rheum. 2008;58(11):3574-3584.

114. Mills R, et al. Intrapulmonary autoantibodies to HSP72 are associated with improved outcomes in IPF. JImmunol Res. 2019;2019:1845128.

115. Heukels P, et al. Enhanced Bruton's tyrosine kinase in B-cells and autoreactive IgA in patients with idiopathic pulmonary fibrosis. Respir Res. 2019;20(1):232.

116. Fernandez IE, Eickelberg O. The impact of TGF- $\beta$ on lung fibrosis: from targeting to biomarkers. Proc Am Thorac Soc. 2012;(3):111-116.

117. Sime PJ, et al. Adenovector-mediated gene transfer of active transforming growth factor- $\beta 1$ induces prolonged severe fibrosis in rat lung. J Clin Invest. 1997;100(4):768-776.

118. Zhao J, et al. Smad3 deficiency attenuates bleomycin-induced pulmonary fibrosis in mice. Am JPhysiol Lung Cell Mol Physiol.2002;282(3):L585-L593.

119 . Coker RK, et al. Transforming growth factors- $\beta 1$, $-\beta 2$, and $-\beta 3$ stimulate fibroblast procollagen production in vitro but are differentially expressed during bleomycin-induced lung fibrosis. Am J Pathol.1997;150(3):981-991.

120. Biernacka A, et al. TGF- $\beta$ signaling in fibrosis. Growth Factors. 2011;29(5):196-202.

121. Shull MM, et al. Targeted disruption of the mouse transforming growth factor- $\beta 1$ gene results in multifocal inflammatory disease. Nature. 1992;359(6397):693-699.

122. Denton CP, et al. Recombinant human anti-transforming growth factor beta1 antibody therapy in systemic sclerosis: a multicenter, randomized, placebo-controlled phase I/II trial of CAT-192. Arthritis Rheum. 2007;56(1):323-333.

123. Rice LM, et al. Fresolimumab treatment decreases biomarkers and improves clinical symptoms in systemic sclerosis patients. J Clin Invest. 2015;125(7):2795-2807. 